\title{
MATHEMATICAL MODELING OF DETONATION INITIATION VIA FLOW CUMULATION EFFECTS
}

\author{
I. Semenov ${ }^{1,2}$, P. Utkin ${ }^{1,2}$, and I. Akhmedyanov ${ }^{1}$ \\ ${ }^{1}$ Institute for Computer Aided Design \\ Russian Academy of Sciences \\ 19/18 Brestskaya 2nd Str., Moscow 123056, Russia \\ ${ }^{2}$ Moscow Institute of Physics and Technology \\ 9 Instituskiy Per., Dolgoprudny 141700, Russia
}

\begin{abstract}
The paper concerns two problems connected with the idea of gaseous detonation initiation via flow cumulation effects and convergence of relatively weak shock waves (SW). The first one is the three-dimensional (3D) numerical investigation of shock-to-detonation transition (SDT) in methane-air mixture in a tube with parabolic contraction followed by the tube section of narrow diameter and conical expansion. The second problem is the numerical study of the start-up of the model small-scale hydrogen electrochemical pulse detonation engine with the use of electrical discharge generating the toroidal SW. The investigation is performed by means of numerical simulation with the use of modern high-performance computing systems.
\end{abstract}

\section{INTRODUCTION}

Theoretical studies of gaseous detonation initiation via flow cumulation effects and convergence of relatively weak SW demonstrates the possibility of significant benefit in energy deposition in comparison with the direct detonation initiation [1]. In authors' findings [2,3], the novel method for SDT due to the special profiling of channel or axisymmetrical tube walls was introduced and investigated. The mechanism of initiation was determined by the gas dynamics cumulation effects when the initiating SW (ISW) interacted with the profiled walls. The stoichiometric propane-air mixture was considered. These results were followed by considering of essentially 3D tube geometries — tube coils [4] and helical tubes [5].

The paper considers two problems. The first one is the 3D numerical investigation of SDT in a stoichiometric methane-air mixture under normal conditions in a tube with parabolic contraction, followed by the tube section of narrow 
diameter and conical expansion. Investigation of critical detonation initiation conditions for natural gas - air mixtures is very important and promising problem from the sense of pulse detonation engine (PDE) development [6].

The second problem is the numerical study of the start-up of the model small-scale hydrogen electrochemical PDE with the use of electrical discharge generating the toroidal SW in the vicinity of detonation chamber (DC) walls. The description of main existing concepts of PDEs can be found in $[6,7]$. The shock-implosion PDE concept implies the use of the imploding SW to initiate a detonation in PDE chamber and the parameters of the engine are discussed in $[8,9]$.

\section{NUMERICAL METHODOLOGY FOR THREE-DIMENSIONAL FLOWS WITH DETONATION WAVES}

\subsection{Mathematical Model}

All calculations are performed with the use of the authors' original software. The system of equations is used describing 3D unsteady flows of a reacting inviscid compressible multispecies gas mixture; in Cartesian frame of reference, this system can be written as

$$
\frac{\partial \mathbf{q}}{\partial t}+\frac{\partial \mathbf{f}_{\mathbf{1}}}{\partial x}+\frac{\partial \mathbf{f}_{\mathbf{2}}}{\partial y}+\frac{\partial \mathbf{f}_{\mathbf{3}}}{\partial z}=\mathbf{S}
$$

where

$$
\begin{gathered}
\mathbf{q}=\left(\begin{array}{c}
\rho \\
\vdots \\
\rho_{N} \\
\rho U_{x} \\
\rho U_{y} \\
\rho U_{z} \\
\rho E
\end{array}\right) ; \quad \mathbf{f}_{\mathbf{1}}=\left(\begin{array}{c}
\rho_{1} U_{x} \\
\vdots \\
\rho_{N} U_{x} \\
\rho U_{x}^{2}+p \\
\rho U_{y} U_{x} \\
\rho U_{z} U_{x} \\
(\rho E+p) U_{x}
\end{array}\right) ; \quad \mathbf{f}_{\mathbf{2}}=\left(\begin{array}{c}
\rho_{1} U_{y} \\
\vdots \\
\rho_{N} U_{y} \\
\rho U_{x} U_{y} \\
\rho U_{y}^{2}+p \\
\rho U_{z} U_{y} \\
(\rho E+p) U_{y}
\end{array}\right) ; \\
\mathbf{f}_{\mathbf{3}}=\left(\begin{array}{c}
\rho_{1} U_{z} \\
\vdots \\
\rho_{N} U_{z} \\
\rho U_{x} U_{z} \\
\rho U_{y} U_{z} \\
\rho U_{z}^{2}+p \\
(\rho E+p) U_{z}
\end{array}\right) ; \quad \mathbf{S}=\left(\begin{array}{c}
\omega_{1} \\
\vdots \\
\omega_{N} \\
0 \\
0 \\
0 \\
0
\end{array}\right) ;
\end{gathered}
$$


$t$ is the time; $x, y$, and $z$ are the Cartesian coordinates; $U_{x}, U_{y}$, and $U_{z}$ are the corresponding velocity components; $\rho, p$, and $E$ are the density, pressure, and total specific energy of the gas mixture; and $\rho_{k}$ and $\omega_{k}$ are the density and density-variation rate due to chemical reactions of the $k$ th species of the mixture. The total specific energy of the gas mixture is determined by the formula:

$E=0.5\left(U_{x}^{2}+U_{y}^{2}+U_{z}^{2}\right)+\sum_{k=1}^{N} \frac{\rho_{k} h_{k}}{\rho}-\frac{p}{\rho} ; \quad h_{k}=h_{0 k}+c_{V k} T+\frac{p_{k}}{\rho_{k}}, k=1, \ldots, N$,

where $p_{k}, h_{k}, h_{0 k}$, and $c_{V k}$ are the partial pressure, specific enthalpy, specific enthalpy of formation, and specific heat at constant volume (which is considered to be constant) of the $k$ th species of the mixture; and $N$ is the number of species in the mixture. Enthalpies of formation and specific heats are taken from [10].

The thermal equation of state of the mixture considered as a perfect gas is:

$$
p=\sum_{k=1}^{N} p_{k}=\sum_{k=1}^{N} \frac{\rho_{k} R T}{\mu_{k}}
$$

where $T$ is the temperature; $R$ is the universal gas constant; and $\mu_{k}$ is the molar mass of the $k$ th species.

For the first problem in consideration, the chemical reactions are modeled by one-stage kinetics of methane combustion:

$$
\mathrm{CH}_{4}+2\left(\mathrm{O}_{2}+3.76 \mathrm{~N}_{2}\right) \rightarrow \mathrm{CO}_{2}+2 \mathrm{H}_{2} \mathrm{O}+7.524 \mathrm{~N}_{2} .
$$

Thus, the number of species $N$ of the gas mixture considered is 5 . They are indexed in the following manner: $\mathrm{CH}_{4}(k=1), \mathrm{O}_{2}(k=2), \mathrm{N}_{2}(k=3)$, $\mathrm{CO}_{2}(k=4)$, and $\mathrm{H}_{2} \mathrm{O}(k=5)$. The density-variation rate for methane is determined as

$$
\omega_{1}=\mu_{1} \psi_{1} ; \quad \psi_{1}=-4 \cdot 10^{14} p^{-1}\left(\frac{\rho_{1}}{\mu_{1}}\right)\left(\frac{\rho_{2}}{\mu_{2}}\right)^{2} \exp \left(-\frac{E^{*}}{R T}\right) \quad[\mathrm{mole} /(\mathrm{l} \cdot \mathrm{s})]
$$

where $\psi_{1}$ is the rate of molar fraction variation; $p$ is the pressure in atm; and $E^{*}=50 \mathrm{kcal} /$ mole. The density-variation rates for the remaining species of the mixture are determined via $\psi_{1}$ and the stoichiometric coefficients in reaction as

$$
\omega_{2}=2 \mu_{2} \psi_{1} ; \quad \omega_{3}=0 ; \quad \omega_{4}=-\mu_{4} \psi_{1} ; \quad \omega_{5}=-2 \mu_{5} \psi_{1} .
$$

The chemical transformations in hydrogen-air mixture for the second problem are described with the detailed kinetic model which includes 17 reversible reactions [11]. The elementary reactions and constants, which determine the speed of forward (subscript $f$ in Table 1) and reverse (subscript $r$ in Table 1) reaction 
Table 1 Parameters of detailed chemical kinetic model for hydrogen oxidation

\begin{tabular}{clccrrrr}
\hline No. & \multicolumn{1}{c}{ Reaction } & $\lg A_{f}$ & $b_{f}$ & \multicolumn{1}{c}{$E_{f}$} & $\lg A_{r}$ & \multicolumn{1}{c}{$b_{r}$} & \multicolumn{1}{c}{$E_{r}$} \\
\hline 1 & $\mathrm{H}+\mathrm{O}_{2} \leftrightarrow \mathrm{OH}+\mathrm{O}$ & 14.27 & 0.0 & 16.79 & 13.17 & 0.0 & 0.68 \\
2 & $\mathrm{O}+\mathrm{H}_{2} \leftrightarrow \mathrm{OH}+\mathrm{H}$ & 10.26 & 1.0 & 8.90 & 9.92 & 1.0 & 6.95 \\
3 & $\mathrm{O}+\mathrm{H}_{2} \mathrm{O} \leftrightarrow \mathrm{OH}+\mathrm{OH}$ & 13.53 & 0.0 & 18.35 & 12.50 & 0.0 & 1.10 \\
4 & $\mathrm{H}_{2} \mathrm{O}+\mathrm{H} \leftrightarrow \mathrm{OH}+\mathrm{H}_{2}$ & 13.98 & 0.0 & 20.30 & 13.34 & 0.0 & 5.15 \\
5 & $\mathrm{H}_{2} \mathrm{O}+M \leftrightarrow \mathrm{H}+\mathrm{OH}+M$ & 16.34 & 0.0 & 105.00 & 23.15 & -2.0 & 0.0 \\
6 & $\mathrm{H}+\mathrm{O}_{2}+M \leftrightarrow \mathrm{HO}_{2}+M$ & 15.22 & 0.0 & -1.00 & 15.36 & 0.0 & 49.9 \\
7 & $\mathrm{HO}_{2}+\mathrm{O} \leftrightarrow \mathrm{OH}+\mathrm{O}_{2}$ & 13.70 & 0.0 & 1.00 & 13.81 & 0.0 & 56.6 \\
8 & $\mathrm{HO}_{2}+\mathrm{H} \leftrightarrow \mathrm{OH}+\mathrm{OH}$ & 14.40 & 0.0 & 1.90 & 13.08 & 0.0 & 40.1 \\
9 & $\mathrm{HO}_{2}+\mathrm{H} \leftrightarrow \mathrm{H}_{2}+\mathrm{O}_{2}$ & 13.40 & 0.0 & 0.70 & 13.74 & 0.0 & 57.8 \\
10 & $\mathrm{HO}_{2}+\mathrm{OH} \leftrightarrow \mathrm{H}_{2} \mathrm{O}+\mathrm{O}_{2}$ & 13.70 & 0.0 & 1.00 & 14.80 & 0.0 & 73.9 \\
11 & $\mathrm{H}_{+} \mathrm{O}+M \leftrightarrow \mathrm{OH}+M$ & 16.00 & 0.0 & 0.00 & 19.90 & -1.0 & 104.0 \\
12 & $\mathrm{O}_{2}+M \leftrightarrow \mathrm{O}+\mathrm{O}+M$ & 15.71 & 0.0 & 115.00 & 15.67 & -0.28 & 0.0 \\
13 & $\mathrm{H}_{2}+M \leftrightarrow \mathrm{H}+\mathrm{H}+M$ & 14.34 & 0.0 & 96.00 & 15.48 & 0.0 & 0.0 \\
14 & $\mathrm{H}_{2} \mathrm{O}_{2}+\mathrm{OH} \leftrightarrow \mathrm{H}_{2} \mathrm{O}+\mathrm{HO}_{2}$ & 13.00 & 0.0 & 1.80 & 13.45 & 0.0 & 32.8 \\
15 & $\mathrm{H}_{2} \mathrm{O}_{2}+\mathrm{O} \mathrm{O}_{2} \leftrightarrow \mathrm{HO}_{2}+\mathrm{HO}_{2}$ & 13.60 & 0.0 & 42.64 & 13.00 & 0.0 & 1.0 \\
16 & $\mathrm{H}_{2} \mathrm{O}_{2}+M \leftrightarrow \mathrm{OH}_{+}+\mathrm{OH}_{+} M$ & 17.08 & 0.0 & 45.50 & 14.96 & 0.0 & -5.1 \\
17 & $\mathrm{H}_{2} \mathrm{O}_{2}+\mathrm{H} \leftrightarrow \mathrm{HO}_{2}+\mathrm{H}_{2}$ & 12.23 & 0.0 & 3.75 & 11.86 & 0.0 & 18.7 \\
\hline & & & & & & &
\end{tabular}

$$
k=A T^{b} \exp \left(-\frac{E}{R T}\right)\left[\left(\mathrm{cm}^{3} / \mathrm{mole}\right)^{m-1} \mathrm{~s}^{-1}\right]
$$

where $m$ is the reaction order, are presented in Table 1.

The rates of molar fraction variation for the components have general notation form for the system of elementary reactions. Component $M$ stands for:

- $\mathrm{N}_{2}$ and $\mathrm{H}_{2} \mathrm{O}$ with collision efficiencies 1.0 and 20.0 in reaction 5;

- $\mathrm{N}_{2}, \mathrm{H}_{2} \mathrm{O}$, and $\mathrm{H}_{2}$ with collision efficiencies 1.0, 21.0, and 3.3 in reaction 6;

- all the components with collision efficiencies 1.0 in reaction 11;

- all the components with collision efficiencies 1.0 in reaction 12;

$-\mathrm{N}_{2}, \mathrm{H}_{2} \mathrm{O}, \mathrm{H}_{2}$, and $\mathrm{H}$ with collision efficiencies 1.0, 6.0, 3.0, and 2.0 in reaction 13 ; and

- $\mathrm{N}_{2}, \mathrm{H}_{2} \mathrm{O}, \mathrm{O}_{2}$, and $\mathrm{H}_{2} \mathrm{O}_{2}$ with collision efficiencies 1.0, 6.0, 0.78, and 6.6 in reaction 16 .

The collision efficiencies are the weights for the individual component rates of molar fraction variation in the expression for the generalized component $M$ rate of molar fraction variation. 


\subsection{Numerical Algorithm}

The method of splitting physical processes is used for the numerical solution of the problem. The system of gasdynamic equations is discretized with respect to spatial variables by means of the finite-volume method. One of the main advantages of the method is its applicability to the unstructured meshes in terms of numerical flux calculation through the computational cell face in the local frame of reference associated with the external normal to the cell face. So, the consideration of computational domains with complex shapes in the global Cartesian frame of reference is possible.

To enhance the accuracy, the MUSCL (Monotonic Upstream-Centered Scheme for Conservation Laws) approach [12] is used with upwind-biased thirdorder (on uniform grids for one-dimensional problems) scheme of interpolation of values at cell centers to faces [13]. The gradient of the solution vector is computed with the moving least squares method [14]. Integration over time is performed by the explicit predictor-corrector scheme with the second-order approximation. The fluxes through the computational cell faces are calculated with Godunov's method [12]. At the final stage, the right-hand source terms connected with chemical reactions are taken into account by solving the corresponding system of ordinary differential equations with the use of backward differentiation formulae.

The numerical algorithm is adapted for investigations to be performed on advanced multiprocessor computational systems with teraflop performance. Parallelization is performed by means of decomposition of a computational domain. For calculations, up to 4000 processor cores were used on Moscow State University supercomputer "Lomonosov."

The detailed information about the numerical procedure for the modeling of multidimensional flows with detonation waves and its parallelization can be found elsewhere [5].

\subsection{Verification}

The chemical kinetic model of methane oxidation was verified in [15] on the basis of the data for self-ignition delays in stoichiometric methane-air mixture obtained in shock-tube experiments and in simulations of two-dimensional (2D) cellular detonation wave (DW) structure in a plane channel.

The chemical kinetic model of hydrogen oxidation was also verified against the data for self-ignition delays in a stoichiometric hydrogen-air mixture obtained in shock-tube experiments [16]. Figure 1 illustrates the comparison of self-ignition delays measured with different procedures with the calculated values based on different kinetic mechanisms. The data from the mechanisms [17-19] are taken 


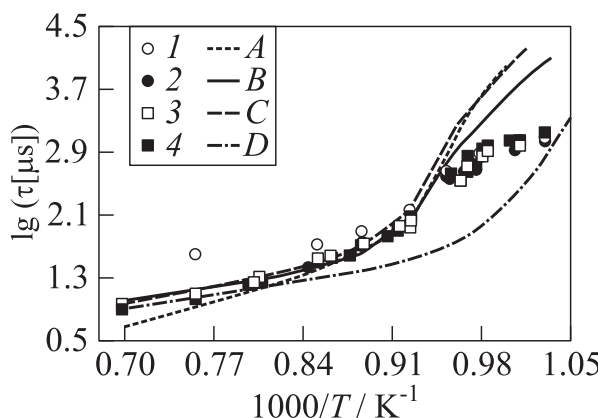

(a)

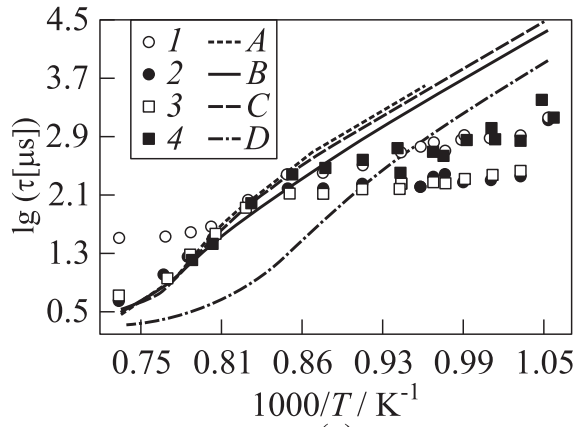

(c)

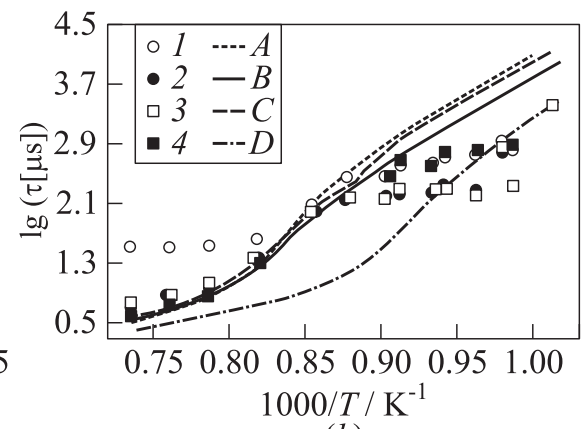

(b)

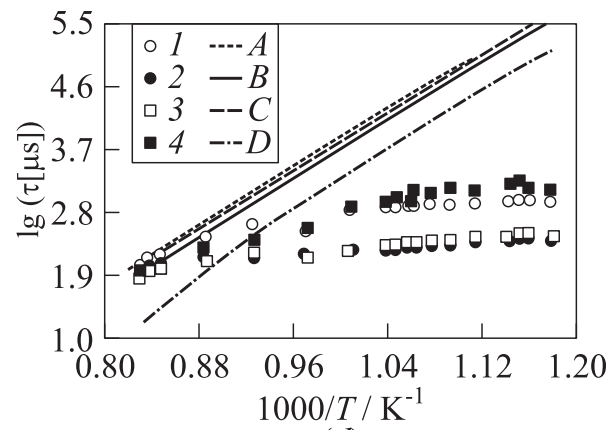

(d)

Figure 1 Comparison of calculated $(A-$ current work, reaction mechanism $[11] ; B-$ [17]; $C-[18] ; D-[19])$ and experimental [16] ( 1 - by luminescence of $\mathrm{OH}$ radical in the axial region; 2 - by luminescence of $\mathrm{OH}$ radical in the near-wall region; 3 by pressure records at the reflecting wall; 4 - by ionization current records ignition delays in a stoichiometric hydrogen-air mixture for different gas densities behind the reflected SW: (a) $0.67 \mathrm{~kg} / \mathrm{m}^{3} ;(b) 1.8 ;(c) 2.8$; and $(d) 4.32 \mathrm{~kg} / \mathrm{m}^{3}$

from [16]. As one can see, the numerical results obtained for the kinetic mechanism [11] qualitatively correspond to the experimental data; for the temperature range 1200-1400 K and densities inherent in problems of detonation initiation and propagation, they correspond quantitatively. However, for the gas temperatures below $1200 \mathrm{~K}$, the calculated ignition delays are significantly greater than the experimental values that could be connected with the reaction mechanism alteration and with the necessity of using other reaction rate constants.

Another test is the modeling of cellular detonation formation in a stoichiometric hydrogen-air mixture under normal conditions in a long plane channel in $2 \mathrm{D}$ statement. The dependency of the detonation cellular structure on the computational cell size is investigated with the aim to determine the most reasonable mesh resolution for the further modeling of a PDE chamber. 
Table 2 Parameters in numerical simulations of detonation cell in hydrogen-air mixture

\begin{tabular}{cccccrr}
\hline $\begin{array}{c}\text { Case } \\
\text { No. }\end{array}$ & $\begin{array}{c}L, \\
\mathrm{~mm}\end{array}$ & $\begin{array}{c}H, \\
\mathrm{~mm}\end{array}$ & $\begin{array}{c}h, \\
\mathrm{~mm}\end{array}$ & $\begin{array}{c}\tau, \\
\mu \mathrm{s}\end{array}$ & \multicolumn{1}{c}{$N_{\text {cell }}$} & \multicolumn{1}{c}{$N_{\text {steps }}$} \\
\hline 1 & 500 & 10 & 0.050 & 0.050 & $2.0 \cdot 10^{6}$ & 50000 \\
2 & 500 & 10 & 0.020 & 0.020 & $12.5 \cdot 10^{6}$ & 125000 \\
3 & 500 & 10 & 0.010 & 0.010 & $50.0 \cdot 10^{6}$ & 250000 \\
4 & 400 & 10 & 0.005 & 0.005 & $160.0 \cdot 10^{6}$ & 400000 \\
\hline
\end{tabular}

The first results on numerical modeling of detonation cells in gaseous mixtures were obtained by the end of the 1970s - early 1980s (refer review and references in [20]). The mathematical models based on the fundamental conservation laws provided the correct reproduction of the complicated dynamic structure of a cellular detonation front involving the leading shock, transverse waves and triple points, and the characteristic cellular pattern formed by the trajectories of triple points. At the same time, a number of articles appear nowadays which focus on further clarification of structure formation; for example, investigation of irregular cells in hydrogen-air detonations [21].

Consider the plane channel with the length $L$ and the width $H$ filled with the quiescent uniform stoichiometric hydrogen-air mixture under normal conditions. Detonation is initiated by several 'discharges' which are modeled by the regions with elevated gas internal energy. All boundary conditions are assumed to be slip walls. The subject of investigation is the evolution of the formed DW. Denote the computational cell size as $h$, the time step as $\tau$, the total number of computational cells as $N_{\text {cell }}$, and the total number of time steps as $N_{\text {steps }}$. It should be noted that in Cases 1-3, the average DW velocity is equal to $2012 \mathrm{~m} / \mathrm{s}$.

Table 2 shows the parameters of the test cases.

Case 1. Numerical calculations for the most coarse computational grid demonstrate the regular cellular pattern (Fig. 2a). The pattern remains the same for the rest part of the channel which is not depicted in Fig. $2 a$. The number of detonation cells per channel height formed at a distance of $0.04 \mathrm{~m}$ from the place of initiation remains constant and equal to 8 . So, the transverse detonation cell size is $1.25 \mathrm{~mm}$ which is too small in comparison with experimental data [22,23].

Case 2. Decreasing the computational cell size for the same channel geometry as in Case 1 leads to the irregular cellular pattern with a larger average detonation cell width (Fig. 2b). The average cell size is estimated as $1.7 \mathrm{~mm}$ (6 detonation cells per channel width) with the instantaneously largest spatial scale of about $3 \mathrm{~mm}$ (see the last snapshot from 0.4 to $0.5 \mathrm{~mm}$ in Fig. $2 b$ ). 
Pressure / $\mathrm{MPa}$
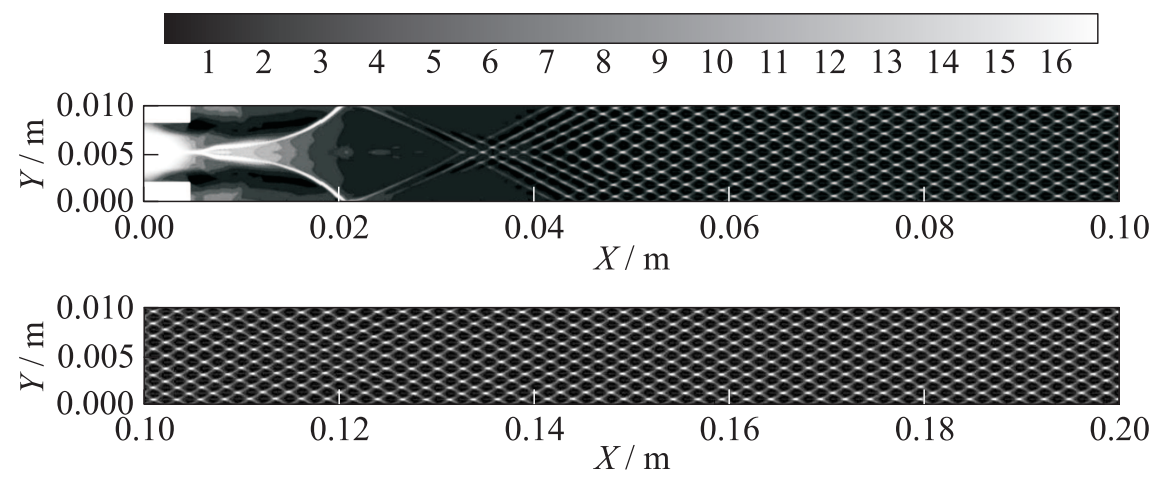

(a)
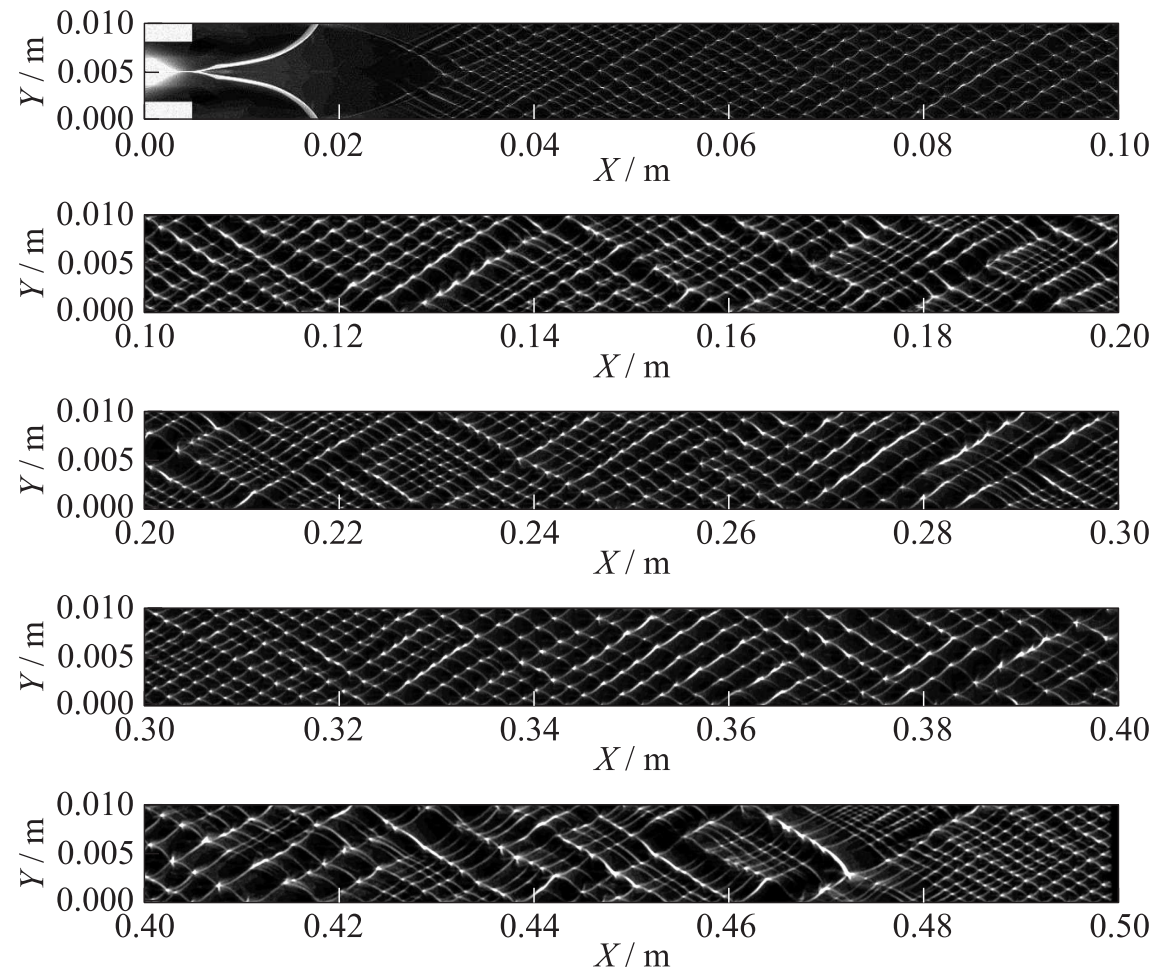

(b)

Figure 2 Numerical soot footprints for Cases $1(a)$ and $2(b)$ 

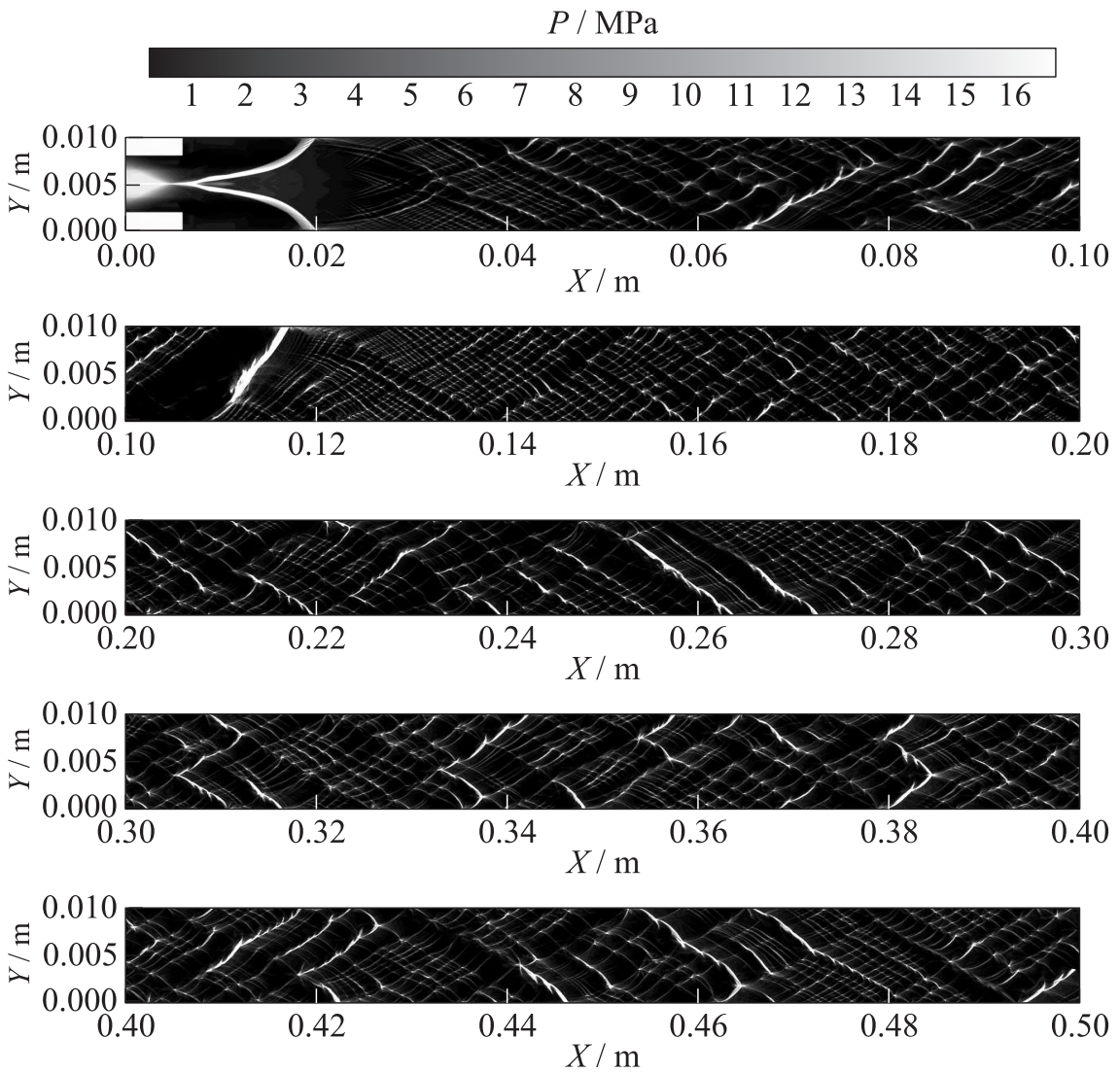

Figure 3 'Numerical soot footprints' for Case 3

Case 3. Further reduction of computational cell size provides highly irregular detonation cellular pattern (Fig. 3). Fine resolution gives the secondary cellular pattern on the primary transverse waves near the place of initiation. It is clear that thick triple point trajectories correspond to the spatial detonation cell scale of several millimeters.

The fruitful concept for explaining the irregular multilevel detonation cell structure has been proposed recently in [24]. Figure 4 illustrates the detonation wave structure at a certain time instant. One can see that thick triple point trajectories form behind the transverse detonations. Inert transverse waves give thin tracks on the cellular pattern.

Case 4. The finest grid corresponds to the computational cell size of $5 \mu \mathrm{m}$. Such resolution provides the secondary cellular structure on transverse detonations but 


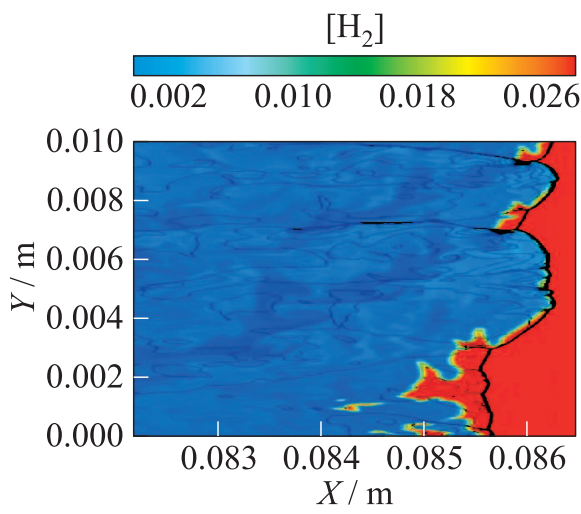

(a)

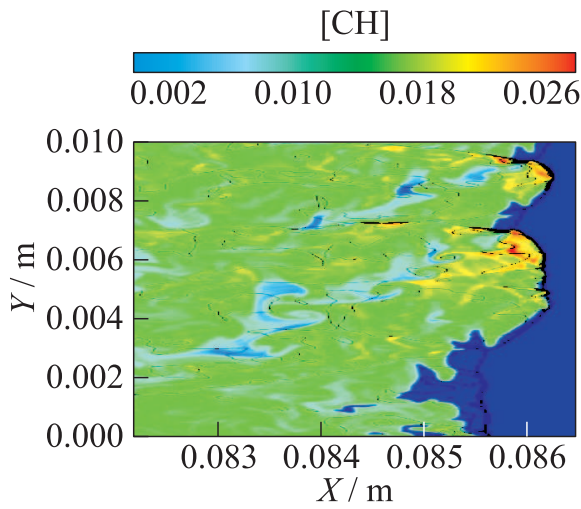

(c)

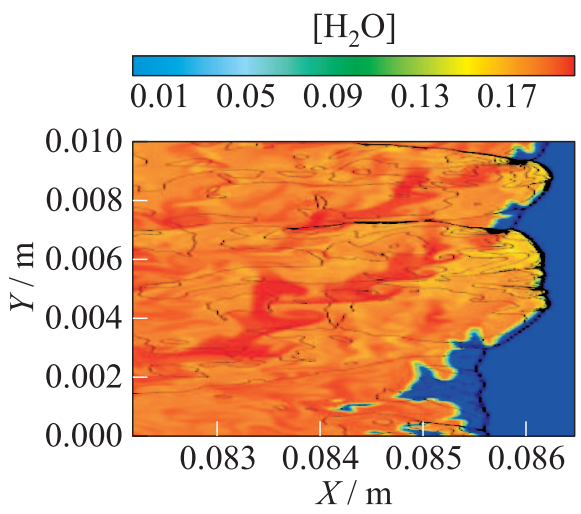

(b)

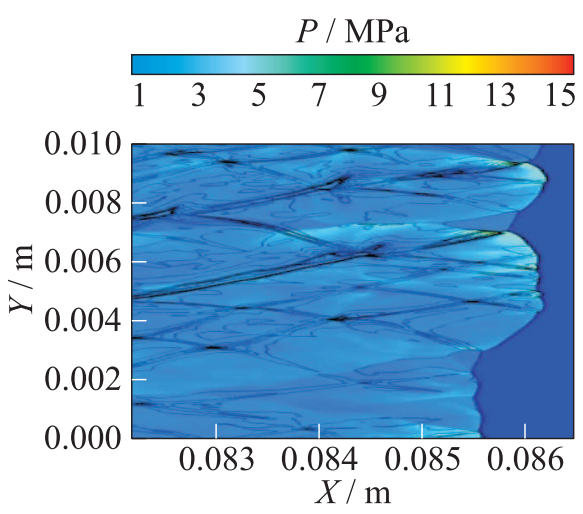

(d)

Figure 4 Typical detonation front structure for Case 3 at some time instant: $(a) \mathrm{H}_{2}$ mass fraction field and pressure isolines; $(b) \mathrm{H}_{2} \mathrm{O}$ mass fraction field and pressure isolines; ( $c$ ) OH mass fraction field and pressure isolines; and $(d)$ gas pressure field and isolines of pressure maximums

also gives the unexpected effect. As one can see in Fig. 5, detonation fails at some distance from the place of initiation. The detonation decay occurs after the formation of one strong transverse detonation wave (thick oblique tracks in the left part of the lower snapshot in Fig. 5) with no detonation recovery up to the maximum run distance of $40 \mathrm{~cm}$ even in a galloping mode. The conclusion can be made that when the grid resolution is good enough to resolve the chemical reaction zones, the channel geometry should satisfy the geometric limits for plane detonation propagation $[25,26]$. In the considered test case, the channel width is too small because the detonation cell size for the stoichiometric hydrogen-air mixture is about $1.5-2 \mathrm{~cm}$. 


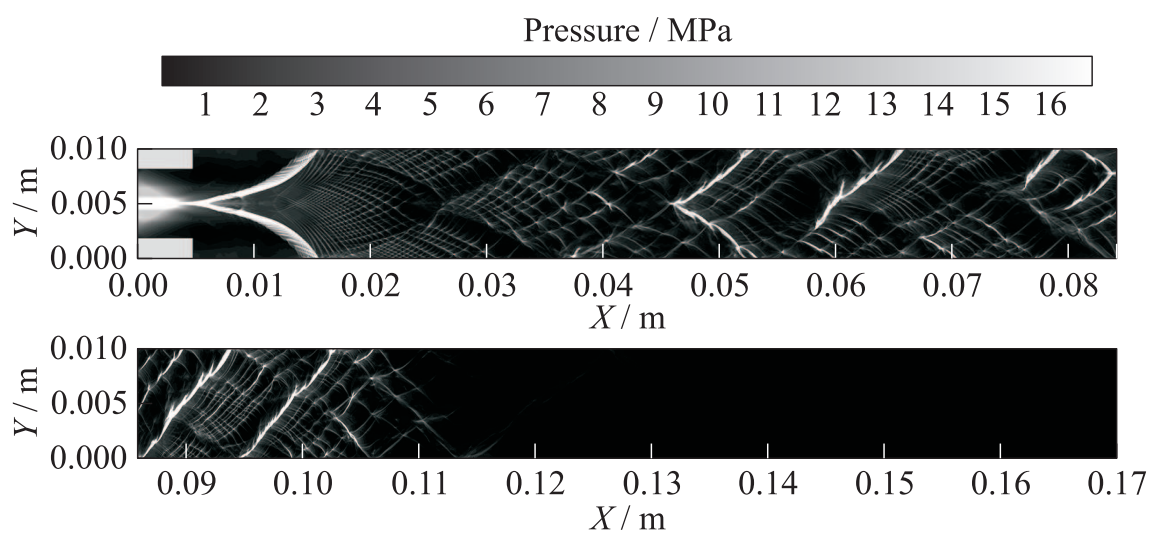

Figure 5 Numerical soot footprints for Case 4

\section{DETONATION INITIATION IN METHANE-AIR MIXTURE}

The round tube of diameter $9.4 \mathrm{~cm}$ comprises five sections: section 1 with constant cross section, parabolic contraction (section 2) connecting narrow section 3 of constant diameter, conical expansion (section 4), and outlet section 5 with constant cross section (Fig. 6). Initially, the tube is filled with the homogeneous, quiescent, stoichiometric methane-air mixture under normal conditions. The tube shape is determined by the geometric parameters from [15].

The motion in the tube is assumed to be initiated by an ISW with zero gradients of parameters directly behind the SW front. Therefore, the boundary condition imposed on the left end of the tube from the initial time to some time $t^{*}$ is the gas inflow with the parameters of the combustible mixture corresponding to the parameters behind an SW with a given Mach number. At time $t^{*}$, the boundary condition is changed to the boundary condition for an inert gas. Other

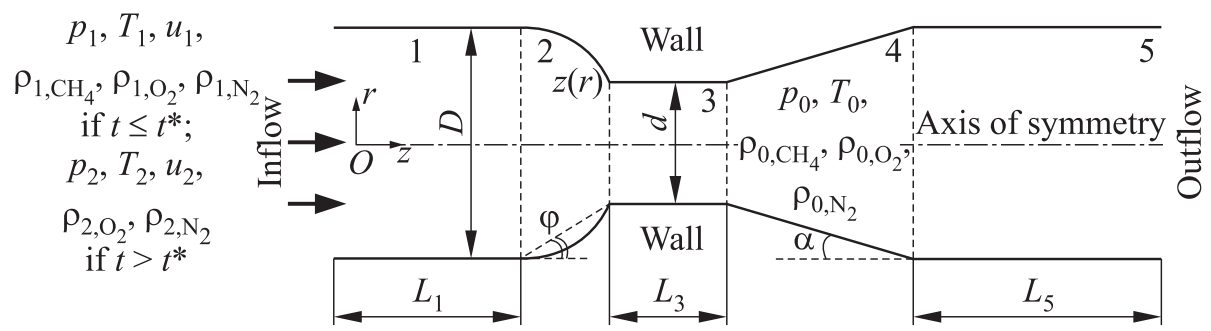

Figure 6 Scheme of the tube with profiled walls (longitudinal cross section) 


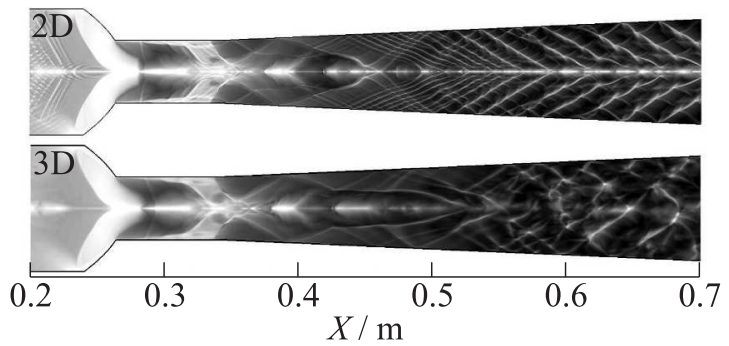

Figure 7 Comparison of numerical soot footprints in 2D and 3D calculations

boundary conditions are the slip condition on the walls and the zero-gradient outflow condition on the right boundary. The calculations are performed up to the time when the disturbances reach the right boundary.

The shape of parabolic contraction and divergence angle of cone expansion are found which provide SDT for the ISW Mach number about 3.3. The results of $3 \mathrm{D}$ numerical investigation confirm in general the results of previous $2 \mathrm{D}$ findings [15]. At the same time, in 3D calculation, the detonation cellular structure is obtained which could not be obtained in $2 \mathrm{D}$ axisymmetrical study (Fig. 7). The possibility of existence of regimes with the DW decaying in conical expansion is demonstrated.

$T / \mathrm{K}$

$300 \quad 85014001950250030503600$

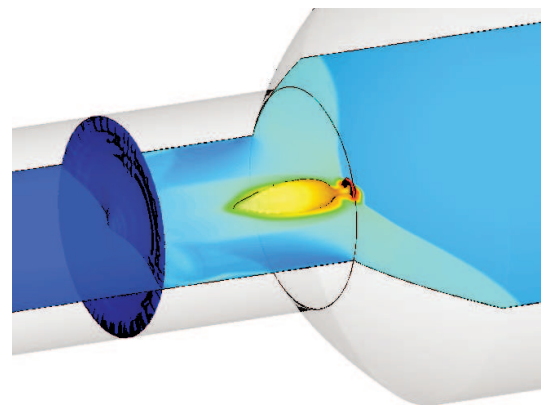

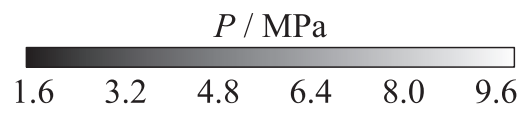

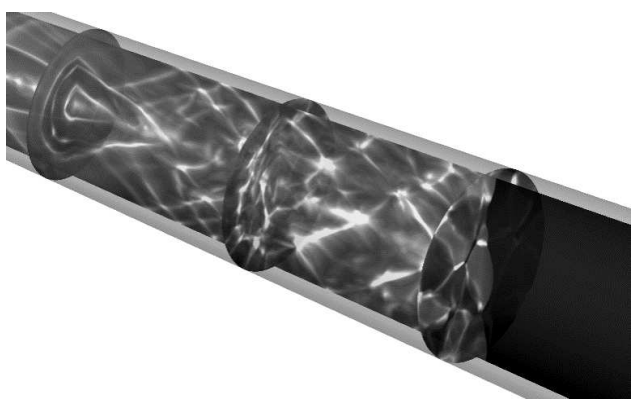

Figure 8 Isosurface of methane den- Figure 9 Numerical soot footprints in sevsity of $0.07 \cdot 10^{-3} \mathrm{~g} / \mathrm{cm}^{3}$ overlapped eral cross sections of conical expansion for the with the gas temperature distribution conditions of DW propagation and predicted gas temperature in longitudinal cross section of the tube 
The computational grid provided spatial resolution from 0.3 to $0.1 \mathrm{~mm}$ and included more than $100 \cdot 10^{6}$ cells. Figure 8 gives an insight to the $3 \mathrm{D}$ pattern of the flow at the instant of local explosion at the symmetry axis. The part of depicted methane density isosurface of yellow color describes the blast wave location and that of blue color - lithotripter SW, of red color - the retonation wave. As one can see in Figs. 7 and 9, in the 3D case, the initially axially symmetric flow becomes $3 \mathrm{D}$ after DW spreads from the connecting section 3 to the conical expansion because of DW front instability. Note that in the 3D statement, the ISW with Much number larger than 3.0 is considered. The additional third degree of freedom in comparison with the 2D statement leads to the instability growth which causes the detonation regime decay in conical expansion for the ISW Mach numbers less than 3.3.

\section{DETONATION INITIATION IN HYDROGEN-AIR MIXTURE IN ELECTROCHEMICAL PULSE DETONATION ENGINE CHAMBER}

The hydrogen electrochemical PDE includes four major parts: inlet 1, detonation chamber 2 , discharge electrodes 3 , and outlet 4 (Fig. 10). In operation, the combustible mixture entering the DC is first ignited by a standard spark plug in the chamber or by the contact with the residual combustion products from the previous cycle. After ignition, the turbulent flame propagates in the DC. When the turbulent flame impinges on electrodes 3 that are connected to an energy storage capacitor, the powerful electric discharge is activated. Electrodes 3 are made with a Rogowsky profile that eliminates electric field enhancement near the electrode edges. The arising 'collar' ring-type electrical discharge creates converging shock waves in the combustible mixture, leading to detonation initiation. The rest of the reactive mixture is burned in the detonation wave that traverses chamber 2 [9].

The computational domain consists of the combustion chamber without inlet and outlet sections of the engine. The computations are performed on a grid with fine spatial resolution of about $0.015-0.02 \mathrm{~mm}$. The total number of cells is about $12.5 \cdot 10^{6}$. The computations are performed in the $3 \mathrm{D}$ domain in the form

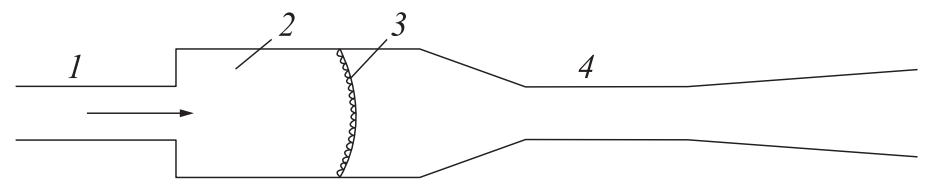

Figure 10 The scheme of electrochemical PDE 
$T / \mathrm{K}$

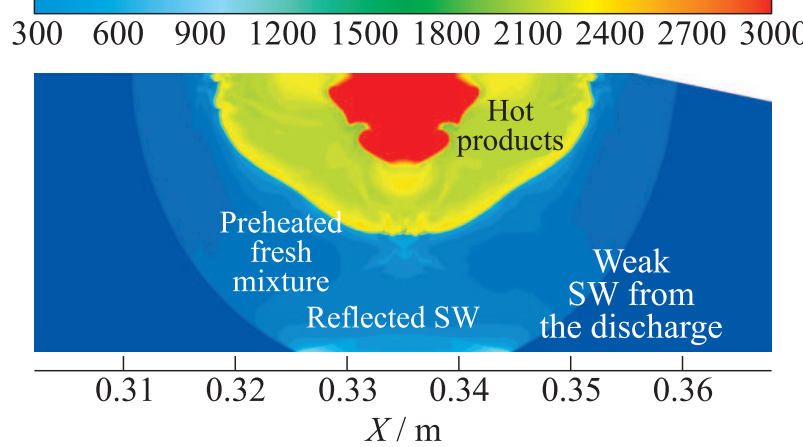

Figure 11 Predicted field of gas temperature for discharge energy $3 \mathrm{~J}$ at time $21 \mu \mathrm{s}$

of a sector with one cell in angular direction. Thus, the simulations are actually $2 \mathrm{D}$ axisymmetrical. The combustion modes arising in chamber are investigated depending on the electrical discharge energy - 3, 6, 7, and $10 \mathrm{~J}$. At the initial time instant, the computational domain is filled with quiescent stoichiometric hydrogen-air mixture under normal conditions. At a distance of $15 \mathrm{~cm}$ from the inlet boundary near the DC wall, the region with elevated pressure and temperature which models the electric discharge is set. The region has a square shape with 2-millimeter edge length. All the calculations are carried out with the constant time step equal to $2 \mathrm{~ns}$.

The operation cycle of the electrochemical PDE is extremely complex because of a lot of interdependent physical and chemical processes. The objective of the current work is to investigate the initial stage of the process, i. e., detonation initiation due to the cumulation of the blast wave from the electrical discharge. Let us describe the combustion modes which occur for the different discharge energies.

It appears that the discharge energy of $3 \mathrm{~J}$ is insufficient to form an SW strong enough to provide a local explosion due to its implosion at the symmetry axis. Figure 11 shows that the ISW from such a discharge initiates chemical reactions behind the front only at the initial stage of the process. The insufficient ISW intensity leads to quenching of chemical reactions. It should be also noted that the blast implosion at the symmetry axis leads to the temperature rise (up to about $900 \mathrm{~K}$ ) which is evidently insufficient for reactive mixture self-ignition. The domain of red color in Fig. 11 corresponds to the gas that initially was placed in the discharge region.

For a discharge energy of $6 \mathrm{~J}$, blast wave cumulation provides local explosion at the DC symmetry axis in contrast to the previous case. However, the strength of this explosion is not sufficient to form the DW and jets of local explosion products spread along the symmetry axis (Fig. 12). 


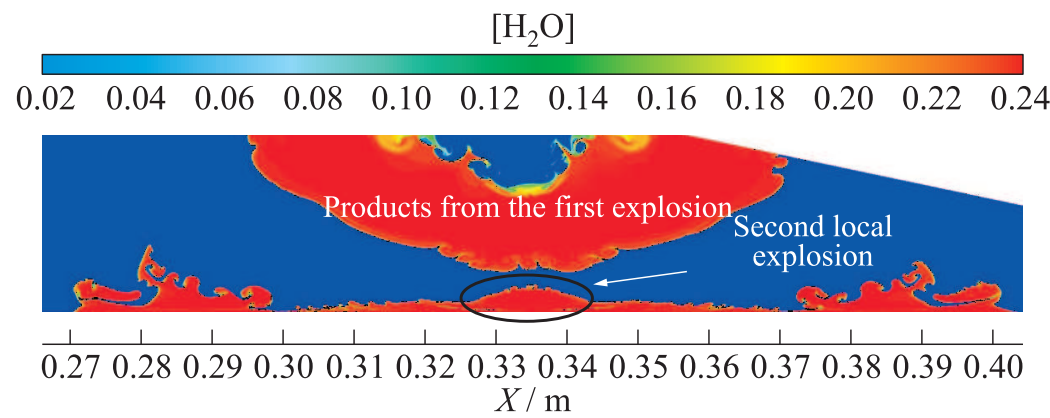

Figure 12 Predicted field of water vapor mass fraction for discharge energy $6 \mathrm{~J}$ at time $72 \mu \mathrm{s}$

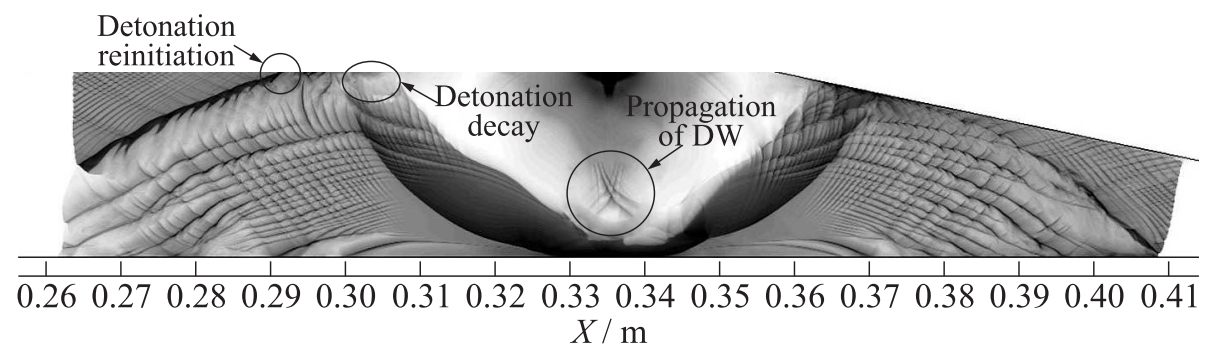

(a)

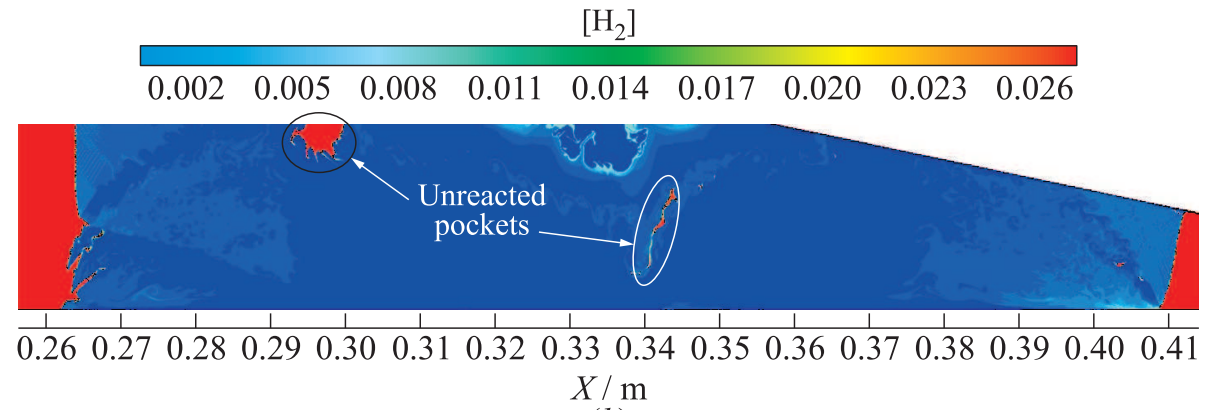

(b)

Figure 13 Process evolution for discharge energy 7 J: $(a)$ numerical soot footprints and (b) hydrogen mass fraction at time $45 \mu \mathrm{s}$

The discharge energy of $7 \mathrm{~J}$ results in the essentially different picture. The analysis of Fig. 13 allows interpreting the process in DC. Observed are here the tracks of triple points directed from the place of discharge to the symmetry axis before the instant of SW focusing. Thus, the discharge energy of $7 \mathrm{~J}$ appears to be sufficient for direct detonation initiation in DC although the DW spreads 


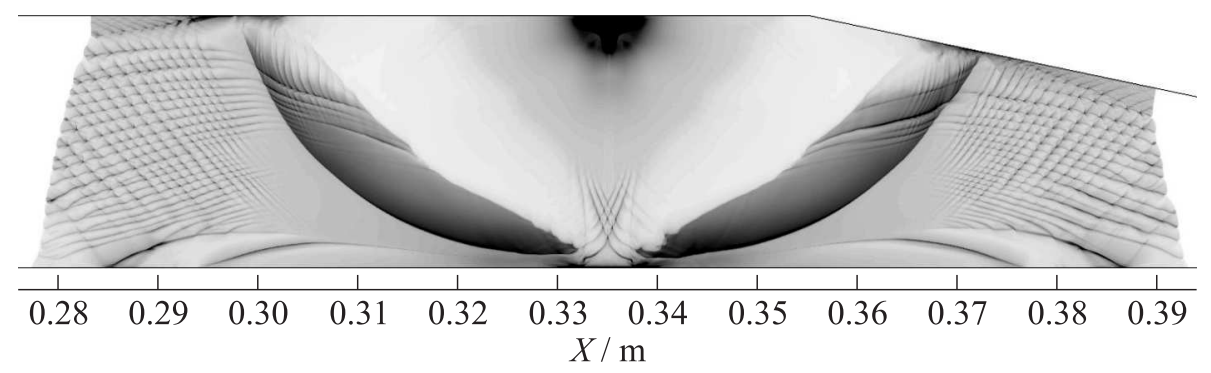

Figure 14 Numerical soot footprints for discharge energy $10 \mathrm{~J}$

not in all directions but only in a narrow region in the direction of symmetry axis. Also, due to the cumulation of the discharge-induced SW at the DC symmetry axis, DWs are formed that spread towards the inlet and outlet sections, as demonstrated by the numerical soot footprints. The other important finding to be noted is the decay of DW propagating from right to left (see the large unreacted pocket of fresh mixture in Fig. 13b) because of geometric expansion and subsequent detonation reinitiation after reflection from the wall.

Further increase in the discharge energy results in better manifestation of all the specific features described above for the case of 7 -joule discharge energy. Soot footprints from the divergent DW get more distinct and vast whereas the detonation cell size gets significantly smaller than the known experimental value because the DW is overdriven (Fig. 14).

\section{CONCLUDING REMARKS}

In the paper, two problems concerning detonation initiation via flow cumulation are investigated. The first one is $3 \mathrm{D}$ numerical investigation of SDT in methane-air mixture in a tube with parabolic contraction followed by the tube section of narrow diameter and conical expansion. The shape of parabolic contraction and divergence angle of cone expansion are found which provide SDT for the ISW Mach number about 3.3. The 3D structure of the formed DW is obtained. The second problem is the numerical study of the start-up of the smallscale hydrogen-fueled electrochemical PDE with the use of electrical discharge producing a toroidal SW. Combustion modes which are realized at different discharge energies are analyzed. The discharge energy of $3 \mathrm{~J}$ is shown to be too small to initiate a local explosion at the DC symmetry axis due to blast wave cumulation. The energy of $6 \mathrm{~J}$ provides a local explosion but DW propagation fails. The energies of $7 \mathrm{~J}$ and higher were shown to be sufficient for engine start-up. The specific features of DW propagation are discussed. 


\section{REFERENCES}

1. Levin, V., S. Osinkin, T. Zhuravskaya, and V. Markov. 2002. Determination of critical conditions for detonation initiation in a finite volume by a converging shock wave. Combust. Explo. Shock Waves 38:693-699.

2. Frolov, S., I. Semenov, P. Komissarov, P. Utkin, and V. Markov. 2007. Reduction of the deflagration-to-detonation transition distance and time in a tube with regular shaped obstacles. Dokl. Phys. Chem. 415:209-213.

3. Semenov, I., P. Utkin, and V. Markov. 2009. Numerical simulation of detonation initiation in a contoured tube. Combust. Explo. Shock Waves 45:700-707.

4. Frolov, S., I. Semenov, I. Akhmedyanov, and V. Markov. 2009. Shock-to-detonation transition in tube coils. 26th Symposium (International) on Shock Waves Proceedings. Springer Verlag. 1:365-370.

5. Semenov, I., I. Akhmedyanov, A. Lebedeva, and P. Utkin. 2011. Three-dimensional numerical simulation of shock and detonation waves propagation in tubes with curved walls. Sci. Tech. Energ. Mat. 72:116-122.

6. Roy, G., S. Frolov, A. Borisov, and D. Netzer. 2004. Pulse detonation propulsion: Challenges, current status, and future perspective. Prog. Energy Combust. Sci. 30:545-672.

7. Wolanski, P. 2011. Detonation engines. J. KONES Powertrain Transport 18:515521.

8. Korobeinikov, V., V. Markov, I. Semenov, P. Pedrow, and S. Wojcicki. 2001. Electrochemical pulse detonation engine. High-speed deflagration and detonation. Moscow: ELEX-KM Publs. 289-302.

9. Korobeinikov, V., V. Markov, I. Semenov, and S. Wojcicki. 2003. About the concept of autocyclomate energy dynamics and the modeling of electrochemical pulsating engine. Russ. J. Chem. Phys. B 22:73-78.

10. McBride, B., S. Gordon, and M. Reno. 1993. Coefficients for calculating thermodynamic and transport properties of individual species. NASA Report TM-4513.

11. Pitz, W., C. Westbrook, W. Proscia, and F. Dryer. 1984. A comprehensive chemical kinetic reaction mechanism for the oxidation of $n$-butane. 20th Symposium (International) on Combustion. 831-843.

12. Toro, E. 2009. Riemann solvers and numerical methods for fluid dynamics. 3rd ed. Springer. $724 \mathrm{p}$.

13. Wesseling, P. 2001. Principles of computational fluid dynamics. Springer. 644 p.

14. Gossler, A. 2001. Moving least squares: A numerical differentiation method for irregularly spaced calculation points. Sandia Report SAND2001-1669.

15. Semenov, I., P. Utkin, I. Akhmedyanov, and N. Demidov. 2012. Multidimensional modeling of detonation initiation in natural gas-air mixtures. 9th ISHPMIE Proceedings. Cracow, Poland. Paper No. 75.

16. Martynenko, V., O. Penyaz'kov, K. Ragotner, and S. Shabunya. 2004. Hightemperature ignition of hydrogen and air at high pressures downstream of the reflected shock wave. J. Eng. Phys. Therm. 77:785-793. 
17. Konnov, A. 1998. Detailed reaction mechanism for small hydrocarbons combustion. Release 0.4. Avalable at: http://homepages.vub.ac.be/ akonnov (accessed March 2008).

18. Konnov, A. 2000. Development and validation of a detailed reaction mechanism for the combustion of small hydrocarbons. 28th Symposium (International) on Combustion. 317.

19. Smith, P., D. Golden, M. Frenklach, N. Moriarty, B. Eiteneer, M. Goldenberg, C. Bowman, R. Hanson, S. Song, W. Gardiner, V. Lissianski, and Z. Qin. GRIMECH 3.0 Reaction Mechanism. Available at: www.me.berkeley.edu/grimech/version30/text30.html (accessed May 13, 2015).

20. Gamezo, V., D. Desbordes, and E. Oran. 1999. Two-dimensional reactive flow dynamics in cellular detonation waves. Shock Waves 9:11-17.

21. Taylor, B., D. Kessler, V. Gamezo, and E. Oran. 2013. Numerical simulations of hydrogen detonations with detailed chemical kinetics. Proc. Combust. Inst. 34:20092016.

22. Bull, D., J. Elsworth, and P. Shuff. 1982. Detonation cell structures in fuel/air mixtures. Combust. Flame 45:7-22.

23. Austin, J. 2003. The role of instability in gaseous detonation. PhD Thesis. California Institute of Technology.

24. Kessler, D., V. Gamezo, and E. Oran. 2011. Multilevel detonation cell structures in methane-air mixtures. Proc. Combust. Inst. 33:2211-2218.

25. Vasiliev, A. 1982. Geometric limits of gas detonation propagation. Combust. Explo. Shock Waves 18:132-136.

26. Guirao, C., R. Knystautas, and J. Lee. 1987. A Summary of hydrogen-air detonation experiments. NUREG/CR-4961, SAND87-7128, R3. 